\title{
Constrictive Pericarditis
}

National Cancer Institute

\section{Source}

National Cancer Institute. Constrictive Pericarditis. NCI Thesaurus. Code C78246.

A heart disorder in which the pericardial sac becomes thickened and fibrotic, tightening the myocardium and impeding the normal myocardial function. 